\title{
Gestión del conocimiento y efectividad organizacional en municipalidades de la provincia de San Martín, Perú
}

\section{Knowledge management and organizational effectiveness in municipalities of the province of San Martín, Peru}

\author{
Recibido: 01 de setiembre $2021 \quad$ Aprobado: 06 de octubre 2021
}

\section{AUTOR 1:}

Santa Cruz Malon

Adan:

Bachiller en Ciencias Administrativas,

Universidad Peruana

Unión, Perú. Trabajador autónomo,

ORCID: 0000-0001-82023684

Correo:

adansantacruz@upeu.edu

.pe

AUTOR 2:

Córdova Rodas_Nelbit Abigail:

Bachiller en Ciencias Administrativas,

Universidad Peruana

Unión, Perú. Trabajador

autónomo,

ORCID: 0000-0003-38445980

Correo:

nelbitcordova@upeu.edu. pe

\section{AUTOR 3:}

Cruz Tarrillo_José Joel:

Magíster en

Administración con

mención en Gestión

Empresarial, Universidad

Peruana Unión, Perú.

Universidad Peruana

Unión,

ORCID: 0000-0002-63725055

Correo:

jose.cruz@upeu.edu.pe

AUTOR 4:

Almestar Villegas_ Carmelino:

Magíster en Investigación y docencia universitaria,

Universidad Peruana

Unión, Perú. Trabajador autónomo,

ORCID: 0000-0002-17578832

Correo:

carmelino.almestar@upeu .edu.pe

\section{RESUMEN:}

La investigación tuvo como propósito determinar la relación entre la gestión del conocimiento (GC) sobre la efectividad organizacional (EO) en el sector público. La muestra estuvo conformada por 220 colaboradores de las municipalidades distritales de Morales, Tarapoto y La Banda de Shilcayo de la región San Martín. Se utilizó dos cuestionarios para recolectar los datos. La validez de los instrumentos se desarrolló mediante juicio de expertos, para lo cual solicitó el análisis de los ítems en cuanto a su claridad, congruencia, contexto y dominio del constructo, por parte de tres expertos; quienes emitieron su valoración sobre la validez de contenido de los cuestionarios. Por otro lado, la confiabilidad de los instrumentos se obtuvo mediante el índice del alfa de Cronbach en una muestra piloto de 15 unidades de estudio. Para la escala de gestión del conocimiento, se obtuvo un alfa de Cronbach de 0,960; mientras que, para la escala de efectividad organizacional, este índice tuvo un valor de 0,924 . Se encontró que las variables gestión del conocimiento y efectividad organizacional se encuentran en un nivel de desarrollo frecuente, es decir las entidades han implementado una adecuada gestión del conocimiento, lo cual trae como consecuencia una adecuada efectividad organizacional; siendo respectivamente sus porcentajes de $50 \%$ y $64 \%$. Por otro lado, se obtuvo una relación media entre ambas variables (Rho: 0.500 y p-valor: 0.000 ).

Palabras clave: desarrollo de competencias, efectividad organizacional, eficiencia, gestión del conocimiento, gestión pública

\section{ABSTRACT:}

The purpose of the research was to determine the relationship between knowledge management $(\mathrm{KM})$ on organizational effectiveness (EO) in the public sector. The sample consisted of 220 collaborators from the district municipalities of Morales, Tarapoto and La Banda de Shilcayo in the San Martín region. Two questionnaires were used to collect the data. The validity of the instruments was developed through expert judgment, for which it requested the analysis of the items regarding their clarity, congruence, context and domain of the construct, by three experts; who gave their assessment on the content validity of the questionnaires. On the other hand, the reliability of the instruments was obtained through the Cronbach's alpha index in a pilot sample of 15 study units. For the knowledge management scale, a Cronbach's alpha of 0.960 was obtained; while for the organizational effectiveness scale, this index had a value of 0.924 . It was found that the variables knowledge management and organizational effectiveness are at a frequent development level, that is, the entities have implemented adequate knowledge management, which results in adequate organizational effectiveness; their percentages being $50 \%$ and $64 \%$ respectively. On the other hand, a mean relationship was obtained between both variables (Rho: 0.500 and p-value: 0.000 ).

Keywords: project, benefit, budget, neighborhoods 


\section{INTRODUCCIÓN}

La gestión del conocimiento es entendida como un proceso sistémico que busca la generación, difusión y adquisición del conocimiento, con la finalidad de lograr la excelencia de las organizaciones, a través del cumplimiento de sus objetivos estratégicos (Correia et al., 2018). Siendo que la gestión del conocimiento es indispensable para lograr la efectividad organizacional; sin embargo, en las entidades públicas del departamento de San Martín, Perú, no existen áreas o personal, dedicado exclusivamente a desarrollas las tareas de gestión del conocimiento como la creación, adquisición, desarrollo, compartición, diseminación, uso, almacenamiento y protección del capital intelectual (conocimiento). De acuerdo con Zelenkov (2018), la gestión del conocimiento organizacional debe desarrollarse simultáneamente con la gestión del cambio, es decir los colaboradores de las municipalidades deben tener flexibilidad para adaptarse a los posibles cambios que puedan surgir en la organización teniendo presente el impacto que buscan alcanzar las municipalidades. Por ello, para que las organizaciones logren la efectividad, es necesario considerar el cambio como un factor crítico, el cual debe basarse en el conocimiento. Sin embargo, es importante resaltar que, los resultados de los modelos de la gestión del conocimiento son más efectivos en las organizaciones privadas que en las públicas, debido a que estas últimas, reproducen el estilo burocrático.

Independientemente de las actividades que desarrollen las organizaciones, es de vital importancia conocer de qué manera se relacionan la gestión del conocimiento con la efectividad organizacional; ya que, a través de la gestión del conocimiento, se busca que los colaboradores tengan un mayor grado de eficiencia al desarrollar sus actividades. Asimismo, en el escenario actual, los cambios cada vez son más constantes; y la flexibilidad de aprender y adaptarse se convierten en una ventaja competitiva en las organizaciones, las cuales se logran al desarrollar la gestión del conocimiento organizacional (Acharya, 2019). Por ello, es importante considerar a la gestión del conocimiento como un elemento crucial el cual permite adquirir competencias en el entorno empresarial, con lo cual se fomenta una cultura del aprendizaje en la organización, y con ello la generación de riqueza (Saad et al., 2020). Asimismo, el conocimiento es un recurso que puede transferirse entre los colaboradores, el cual se basa en la utilización de la información, con la finalidad de solucionar problemas y promover la obtención de resultados (Passaillaigue and Estrada, 2016). En ese sentido, se considera como funciones de la gestión del conocimiento, el almacenamiento, la transferencia y la aplicación del conocimiento en las empresas. 
Kumar (2017), por ello, es crucial resaltar la importancia que tienen los modelos de gestión del conocimiento para las empresas se debe a la consideración que se le da al conocimiento como un recurso estratégico para lograr la competitividad (Corrêa, Falsarella, and Cibele, 2016).

Con respecto al estado del arte, Saad et al. (2020) desarrollaron un estudio para conocer las prácticas de gestión del conocimiento y características de liderazgo transformacional en industrias de bienes de consumo; por otro lado, Zelenkov (2018) desarrolló un estudio para conocer el impacto de la gestión del conocimiento y la preparación para el cambio en la eficacia de las organizaciones estatales y privadas rusas; sin embargo no se han realizado estudios para conocer la relación que existe entre la gestión del conocimiento y la efectividad organizacional en entidades del sector público; por ello es importante desarrollar el presente estudio, debido a que se ha identificado una laguna del conocimiento.

De igual modo, es importante que la empresa deba medir la efectividad organizacional del cumplimiento de los objetivos organizacionales, trazados a diferentes plazos, para lo cual se analizará tanto la eficiencia como la eficacia, a través del uso de indicadores; por ello es necesario que la alta dirección tome en cuenta la efectividad organizacional, considerando tanto los resultados como la capacidad de producción de los colaboradores (Corrêa et al., 2016).

En el presente estudio, se pretende cerrar esta brecha del conocimiento mediante el análisis de la relación entre la gestión del conocimiento y la efectividad organizacional en entidades del sector público. Los resultados del estudio permitirán determinar en qué medida están asociadas estas variables de tal manera que se puedan tomar acciones para mejorar la afectividad, a través de una mejor gestión del conocimiento.

El objetivo del estudio fue determinar la relación entre la gestión del conocimiento y la efectividad organizacional en entidades del sector público, en la provincia de San Martín, departamento del mismo nombre, en el Perú. Asimismo, se planteó la siguiente hipótesis: existe relación significativa entre la gestión del conocimiento y la efectividad organizacional en entidades del sector público.

\section{MARCO TEORICO /DESARROLLO}

\subsection{Gestión del conocimiento}

La teoría de la creación del conocimiento organizacional presentó amplia aceptación e impacto tanto en los medios académicos como profesionales, cuando se publicó hace más de dos décadas por los investigadores Nonaka y Takeuchi publicaron un estudio 
en 1994, sobre la creación del conocimiento organizacional, en dicho trabajo indican que el conocimiento que puede ser expresado en palabras y números, representa solamente el extremo del iceberg del conjunto de conocimientos como un todo, de tal manera que las organizaciones deben buscar medios que propicien la generación de nuevos conocimientos identificando elementos para una mejor gestión de sus procesos (Bandera et al., 2017). Estos autores proponen como teoría la gestión del conocimiento en su dimensión epistemológica, en la cual existe el conocimiento tácito y el conocimiento explícito; el primero de ellos está en el individuo, mientras que el segundo es el resultado de la conversión y amplificación del primero, pasando a la organización en forma de espiral (Roza, 2020).

Asimismo, la teoría del conocimiento organizacional, fue propuesta por Nonaka; en la cual se describe como el conocimiento organizacional es creado por los individuos y amplificado por las relaciones humanas; por ello esta teoría valoriza las relaciones humanas, las cuales son estimuladas por los facilitadores de la gestión del conocimiento (Xavier, Oliveira, and Teixeira, 2012). Por otro lado, la gestión del conocimiento se define como un proceso sistémico, a través del cual, se genera, codifica, difunde y se adquiere el conocimiento, buscando el logro de la excelencia empresarial (Correia et al., 2018).

Las etapas de generación del conocimiento en las empresas, se presentan en una serie de interacciones entre el conocimiento tácito y el explícito. El conocimiento tácito se socializa en la organización desde sus clientes y otros grupos de interés. Este conocimiento se procesa iterativamente a través de procesos a medida que el conocimiento tácito se articula; es decir, se transforma en explícito, y se combina para respaldar la salida de un producto o servicio de la organización. En este sentido, la externalización es el proceso por el cual el conocimiento tácito se transforma en conocimiento explícito y la internalización es el proceso inverso (Koehler, Caetano, and Rogério, 2019).

Se considera como dimensiones de la GC a la cultura organizacional, comunicación interna, desarrollo de competencias y planificación. Se define cultura organizacional como un nuevo enfoque que posibilita a la gerencia comprender mejor a la organización. La cultura organizacional es un sistema dinámico de la empresa, ya que los valores empresariales pueden modificarse; asimismo, la comunicación interna, es un recurso gerencial que se utiliza como herramienta para generar nuevas competencias en los colaboradores; el desarrollo de competencias, se definen como las características relacionadas con la actuación exitosa de los trabajadores. Asimismo, se definen como hábitos de conducta que hacen posible trabajar mejor al colaborador; y la planificación, está representada por las actividades consideradas con una visión de futuro, 
considerando los medios y recursos para lograr las metas deseadas (Mauricio, Pailaquilén, and Medina, 2019).

Sin embargo, existen diversos enfoques sobre la concepción de la gestión del conocimiento organizacional, debido a que es un término complejo y abstracto, por esta razón se generan debates continuos entre filósofos en el campo de la epistemología (Gao, Chai, and Liu, 2018).

\subsection{Efectividad organizacional}

La efectividad organizacional, se define como el logro de la eficacia y la eficiencia, la primera se cumple cuando se alcanza los objetivos, mientras que la segunda, se define como el uso óptimo de los recursos (Marín and Cuartas, 2019), A lo largo de los años ha habido diversos modelos sobre la efectividad organizacional junto con diversos indicadores que permiten medir la efectividad; siendo cuatro los modelos más importantes que definen la efectividad organizacional enfoque en las metas, enfoque en los recursos del sistema, modelo de procesos y modelo de grupos estratégicos (Ashraf and Abd, 2012). La efectividad organizacional es un término polifacético que abarca diversos componentes para medir el desempeño organizacional; asimismo la efectividad organizacional se define como un componente del desempeño de la organización; de igual manera se entiende como la capacidad que tiene a largo plazo para lograr de forma coherente sus objetivos estratégicos y operativos (Kataria, Rastogi, and Garg, 2012).

Se define a la efectividad de una organización como la medida con la cual se logran las metas propuestas; de igual modo la efectividad organizacional es un factor que mide qué tan bien una organización logra sus objetivos, tanto en organizaciones con fines de lucro como en organizaciones sin fines de lucro (Rehman et al., 2019).

Aunque ha sido difícil conceptualizar con exactitud a la efectividad organizacional de manera amplia, una definición consensuada consiste en la medida en que una organización logra sus metas; debido a su carácter multidimensional, una organización puede ser juzgada simultáneamente efectiva por un criterio e ineficaz por otro (Kataria et al., 2012). Igualmente, la efectividad organizacional resulta de ajustar las características de la organización a factores clave, relacionados con los desafíos particulares que enfrenta la organización, de igual modo, la efectividad organizacional mejora cuando se aumenta el conocimiento de los colaboradores, haciéndolos menos dependiente de otros; por ello cuanto mayor sea el grado de integración del conocimiento requerido para lograr la efectividad, mayor será la necesidad de coordinar el proceso general de aplicación del conocimiento a través de una secuencia bien diseñada de trabajo cooperativo (Victer, 2020). 
Por otro lado, la efectividad organizacional, está compuesta por tres factores: cumplimiento de expectativas, satisfacción del equipo y el logro de compromisos (Ariza, 2017). Mediante el cumplimiento de estas dimensiones, la empresa tendría una ventaja competitiva frente a la competencia. Asimismo, para Camue et al. (2017), la efectividad organizacional, está conformada por la eficiencia y la eficacia empresarial.

Las dimensiones de la efectividad organizacional son: eficiencia, eficacia, flexibilidad y desarrollo de estrategias. A continuación, se definen de acuerdo con (Zidane and Olsson, 2017): (a) Eficiencia, relación entre resultados logrados y recursos utilizados; (b) eficacia, se alcanza con el logro de objetivos planificados; (c) flexibilidad, se define como la adaptación de la empresa a las necesidades del mercado; y (c) desarrollo de estrategias, se define como el modo de dar forma explícita a las metas y objetivos organizacionales a largo plazo. La estructura que se siguió para desarrollar el estudio fue: en primer lugar, se presenta la introducción, en la cual se describe el problema de investigación, luego se describe el marco teórico que sustenta las variables, en tercer lugar, se describe el método para la recolección de los datos, luego se presentan los resultados y la discusión, finalmente se muestran las conclusiones del estudio.

\section{MÉTODO}

Para el desarrollo del estudio se utilizó un diseño no experimental transversal y tipo de investigación correlacional, ya que no se manipuló ninguna variable, únicamente se recolectó los datos tal como se presentan naturalmente en el acontecer de las unidades de estudio. Asimismo, es de corte transversal porque las mediciones se obtendrán por única vez. Por otro lado, los estudios relacionales buscan determinar el nivel de asociación entre variables. Para determinar la relación entre las variables se utilizó el coeficiente Rho de Spearman con un 95\% de confianza.

Se consideró como población a la cantidad de trabajadores de las municipalidades de Morales, La Banda de Shilcayo y Tarapoto, como se muestra en la tabla 1. El tamaño de la población es 220 colaboradores de las municipalidades distritales de Morales, Tarapoto y La Banda de Shilcayo, de la provincia de San Martín, región del mismo nombre en el Perú. Se consideró únicamente al personal administrativo de la modalidad nombrado o permanente.

Tabla 1

Distribución de colaboradores, según municipalidad distrital

\begin{tabular}{lcc}
\hline Municipalidad & N & Porcentaje \\
\hline Morales & 30 & 13 \\
La Banda de Shilcayo & 43 & 20 \\
Tarapoto & 147 & 67 \\
Total & 220 & 100 \\
\hline
\end{tabular}


Los criterios de inclusión para la unidad de estudio son los colaboradores que se encuentren laborando en cada una de las municipalidades de Morales, La Banda de Shilcayo y Tarapoto de la provincia. Por otro lado, los criterios de exclusión para la unidad de estudio, son los colaboradores que debido a su salud no participaron del estudio.

Las variables del presente estudio son la gestión del conocimiento y efectividad organizacional, elaborados por Velásquez (2018). La gestión del conocimiento se define como un proceso sistemático, articulado e intencional; apoyado en la generación, codificación, diseminación y apropiación de conocimientos, con el propósito de alcanzar la excelencia organizacional (Correia et al., 2018). Por otro lado, se define como la concomitancia entre eficacia y eficiencia. La eficacia es el logro de objetivos y la eficiencia es el uso óptimo de los recursos (Marín and Cuartas, 2019).

Se utilizó como técnica a la encuesta, la cual consiste en la aplicación de cuestionarios a las unidades de estudio. Asimismo, se utilizó dos cuestionarios que evalúan la gestión del conocimiento y efectividad organizacional. La validez de los instrumentos se desarrolló mediante juicio de expertos, para lo cual solicitó el análisis de los ítems en cuanto a su claridad, congruencia, contexto y dominio del constructo, por parte de tres expertos; quienes emitieron su valoración sobre la validez de contenido de los cuestionarios. Por otro lado, la confiabilidad de los instrumentos se obtuvo mediante el índice del alfa de Cronbach en una muestra piloto de 15 unidades de estudio. Para la escala de gestión del conocimiento, se obtuvo un alfa de Cronbach de 0,960; mientras que, para la escala de efectividad organizacional, este índice tuvo un valor de 0,924. Estos valores muestran una confiabilidad elevada para ambos instrumentos.

\section{RESULTADOS.}

\subsection{Análisis descriptivo}

Se encontró que, la gestión del conocimiento en las municipalidades distritales de Morales, Tarapoto y La Banda de Shilcayo, se practica en un nivel casi siempre (50\%), es decir estos colaboradores del sector público crean y comparten conocimiento corporativo para alcanzar los objetivos organizacionales, ya que la gestión del conocimiento, permite generar nuevos conocimientos que agregan valor a los servicios prestados a la sociedad, por medio de una mayor colaboración entre los servidores y el acceso simplificado a fuentes de información y aprendizaje por parte de los colaboradores. 
Por otro lado, con respecto a las dimensiones, la cultura organizacional se viene practicando siempre $(71 \%)$, lo que significa que los valores y creencias de la organización se comparten al interior de la administración de las municipalidades; por otro lado, la comunicación interna se practica siempre (42\%), lo cual es vital para compartir el conocimiento tácito de la organización; el desarrollo de competencias en las municipalidades se viene realizando siempre (51\%); es decir los colaboradores están adquiriendo conocimientos a través de la educación corporativa, coaching y mentoring; y la planificación se practica siempre (57\%); con lo cual se programa los contenidos que serán suministrados a los trabajadores de las diversas áreas de las municipalidades; a través de la educación corporativa.

Tabla 2

Análisis descriptivo de la gestión del conocimiento

\begin{tabular}{|c|c|c|c|}
\hline Variable/dimensión & Nivel & Frecuencia & Porcentaje \\
\hline \multirow{6}{*}{ Gestión del conocimiento } & Nunca & 4 & 2 \\
\hline & Casi nunca & 7 & 3 \\
\hline & A veces & 21 & 10 \\
\hline & Casi siempre & 78 & 35 \\
\hline & Siempre & 110 & 50 \\
\hline & Total & 220 & 100 \\
\hline \multirow{6}{*}{ Cultura organizacional } & Nunca & 1 & 1 \\
\hline & Casi nunca & 6 & 3 \\
\hline & A veces & 14 & 6 \\
\hline & Casi siempre & 41 & 19 \\
\hline & Siempre & 158 & 71 \\
\hline & Total & 220 & 100 \\
\hline \multirow{6}{*}{ Comunicación interna } & Nunca & 5 & 2 \\
\hline & Casi nunca & 9 & 4 \\
\hline & A veces & 33 & 15 \\
\hline & Casi siempre & 81 & 37 \\
\hline & Siempre & 92 & 42 \\
\hline & Total & 220 & 100 \\
\hline \multirow{6}{*}{ Desarrollo de competencias } & Nunca & 6 & 3 \\
\hline & Casi nunca & 5 & 2 \\
\hline & A veces & 24 & 11 \\
\hline & Casi siempre & 73 & 33 \\
\hline & Siempre & 112 & 51 \\
\hline & Total & 220 & 100 \\
\hline \multirow{6}{*}{ Planificación } & Nunca & 2 & 1 \\
\hline & Casi nunca & 9 & 4 \\
\hline & A veces & 20 & 9 \\
\hline & Casi siempre & 64 & 29 \\
\hline & Siempre & 125 & 57 \\
\hline & Total & 220 & 100 \\
\hline
\end{tabular}

Fuente elaborado por el autor.

Asimismo, en la tabla 3 se describe a la efectividad organizacional de las municipalidades de Morales, Tarapoto y La Banda de Shilcayo, la cual se desarrolla en un nivel siempre (64\%), es decir se tiene un impacto medio de las acciones que desarrollan los colaboradores de las municipalidades, sobre la población para cambiar 
la realidad. Asimismo, la eficiencia se desarrolla en las municipalidades, en un nivel casi siempre (44\%), o sea, se desarrollan buenas prácticas sobre el uso óptimo de los recursos. Por otro lado, la eficacia se desarrolla siempre (61\%), por parte de los colaboradores de las municipalidades, lo cual significa que se está alcanzando los resultados y metas de manera satisfactoria. En cuanto a la flexibilidad se presenta en un nivel de siempre (76\%), es decir, los colaboradores son capaces de adaptarse a los resultados que persigue la organización, mientras que, el desarrollo de estrategias alcanzó un nivel predominante de siempre (59\%), esto quiere decir que, los colaboradores de las municipalidades, participan en el planteamiento de estrategias, juntamente con la alta dirección para alcanzar sus objetivos organizacionales.

Tabla 3

Análisis descriptivo de la efectividad organizacional

\begin{tabular}{lccc}
\hline Variable/dimensión & Nivel & Frecuencia & Porcentaje \\
\hline \multirow{4}{*}{ Efectividad organizacional } & A veces & 9 & 4 \\
& Casi siempre & 70 & 32 \\
& Siempre & 141 & 64 \\
& Total & 220 & 100 \\
& Casi nunca & 1 & 1 \\
Eficiencia & A veces & 36 & 16 \\
& Casi siempre & 98 & 44 \\
& Siempre & 85 & 39 \\
& Total & 220 & 100 \\
Eficacia & Casi nunca & 2 & 1 \\
& A veces & 14 & 6 \\
& Casi siempre & 70 & 32 \\
& Siempre & 134 & 61 \\
Flexibilidad & Total & 220 & 100 \\
& A veces & 9 & 4 \\
& Casi siempre & 44 & 20 \\
& Siempre & 167 & 76 \\
Desarrollo de estrategias & Total & 220 & 100 \\
& A veces & 9 & 4 \\
& Casi siempre & 81 & 37 \\
& Siempre & 130 & 59 \\
& Total & 220 & 100 \\
\hline
\end{tabular}

\subsection{Análisis relacional}

Se obtuvo una relación media positiva (Rho:0.500) entre la efectividad organizacional y la gestión del conocimiento. En la tabla 4 también se muestra la relación entre EO y las dimensiones de GC. Esto significa que, la GC tienen una relación de grado medio con la EO. 
Tabla 4

Análisis de correlación

\begin{tabular}{lccc}
\hline Variable/dimensión & Rho Spearman & p-valor & $\mathrm{N}$ \\
\hline Cultura organizacional &, 243 & .000 & 220 \\
Comunicación interna &, 417 & .000 & 220 \\
Desarrollo de competencias &, 445 & .000 & 220 \\
Planificación &, 438 & .000 & 220 \\
Gestión del conocimiento &, 500 & .000 & 220 \\
\hline
\end{tabular}

\section{DISCUSIÓN}

Se obtuvo una asociación positiva media (Rho: 0.500 ) entre la GC y la EO, con un pvalor de 0.000; es decir existe una asociación de grado medio, por ello, al aumentar el nivel de gestión del conocimiento, se espera obtener un nivel medio de efectividad organizacional en los trabajadores de las municipalidades; es decir al mejorar el nivel de gestión del conocimiento a través de la identificación, creación, adquisición, desarrollo, compartición, diseminación, uso, almacenamiento y protección del capital intelectual (conocimiento), se logrará mejorar el indicador de impacto de las municipalidades, es decir se cambiará la realidad (brechas sociales) de la población a la cual presta servicios la municipalidad. Esta afirmación es respaldada por Tang (2017) en Shanghai, quienes encontraron una relación positiva entre la GC y EO, en los colaboradores de un hospital de Shanghai, es decir ambas variables están relacionadas. Sustentando los hallazgos, Passaillaigue \& Estrada (2016), mencionan que, la gestión del conocimiento es una estrategia organizacional que juega un papel muy importante para obtener ventajas competitivas en la organización. Asimismo, concluyen que para el desarrollo de una adecuada GC se debe tener en cuenta tres elementos: las personas, los medios y las tecnologías. Corrêa et al. (2016), señalan que las organizaciones que adoptan un modelo de gestión del conocimiento, tienden a innovar en sus procesos, productos y servicios; mejorando de esta manera su indicadores de impacto como es la efectividad.

Concerniente a la efectividad organizacional, Camue, Carballal, \& Toscano (2017), afirman que, para lograr este indicador de impacto, la empresa debe buscar el equilibrio entre los objetivos, el uso de recursos y la satisfacción de los colaboradores. Por ello, Khan \& Vorley (2017), sostienen que los modelos de GC implementados en las organizaciones, promueven el desarrollo de ventajas competitivas, logrando con ello la efectividad organizacional. Para Abdi et al., (2018), la GC además se relaciona con la innovación de las empresas, trayendo consigo, ventajas competitivas para las organizaciones, mejorando sus indicadores de desempeño.

Los hallazgos del presente estudio, permitirán que los responsables políticos y administradores de las municipalidades, puedan formular políticas y estrategias de 
innovación para alcanzar ventajas competitivas. De otro lado, Bustinza et al. (2019), sostienen que, para que las empresas puedan mejorar su eficacia organizacional, es necesario que las organizaciones y sus miembros actúen con resiliencia, cuando las circunstancias cambien negativamente, esto significa tener resiliencia en todos los niveles de la organización. Por ello, las organizaciones deben adquirir y saber cómo crear, acumular, compartir y transferir conocimiento con todas las partes interesadas; es decir las municipalidades deben identificar el conocimiento que poseen sus colaboradores, para luego utilizarlo en el logro de sus objetivos y metas, a saber, la satisfacción de necesidades sociales.

De otro lado, Fashami \& Babaei (2017), indican que la gestión del conocimiento, tendrá efectividad, cuando se tome en cuenta las siguientes dimensiones: liderazgo transformacional, habilidades humanas y sociales, orientación al conocimiento, inteligencia emocional y el clima de confianza entre los colaboradores; tanto de los colaboradores como del nivel estratégico. Para Alimehr et al., (2019), la gestión basada en el conocimiento permite empoderar a los empleados y de esta manera mejorar la eficiencia y eficacia de la organización; por ello es indispensable que las municipalidades distritales de la provincia de San Martín no tengan acciones punitivas hacia los colaboradores que busquen socializar su conocimiento tácito. De otro lado, es importante considerar el enfoque sistémico, con lo cual se permitirá el surgimiento de nuevas posibilidades y potencialidades de los colaboradores, al interior de las organizaciones.

Finalmente, Zeña \& Arévalo (2016), señalan que el enfoque de la GC promueve el compromiso y la colaboración reflexiva y activa de los colaboradores, mediante la armonía de ideas eficaces y efectivas buscando siempre el logro de los objetivos organizacionales.

\section{CONCLUSIONES.}

Basado en el presente estudio, se concluye que la gestión del conocimiento y la efectividad organizacional de las municipalidades del departamento de San Martín, se relacionan en un grado medio; es decir la cultura organizacional, la comunicación interna, el desarrollo de competencias y la planificación tienen un impacto en la efectividad organizaciones de las organizaciones en estudio. Asimismo, la gestión del conocimiento es el inicio para que las organizaciones del sector público socialicen, articulen y combinen el conocimiento, pasando del conocimiento tácito al implícito; y de esta manera se alcance niveles aceptables de efectividad organizacional. 
Asimismo, entre las limitaciones del estudio, se tienen: No se abarcó a toda la población de trabajadores de las tres municipalidades, debido a que algunos de ellos desarrollaban sus actividades laborales en forma virtual por la pandemia de la COVID 19. Otra limitación de la investigación se debe a que no se consideró a todas las municipalidades distritales de la provincia de San Martín, por lo cual no se puede generalizar los hallazgos para otras municipalidades de la mencionada provincia.

\section{REFERENCIAS BIBLIOGRÁFICAS}

Abdi, K., Mardani, A., Senin, A. A., Tupenaite, L., Naimaviciene, J., Kanapeckiene, L., and Kutut, V. (2018). The effect of knowledge management, organizational culture and organizational learning on innovation in automotive industry. Journal of Business Economics and Management, 19(1), 1-19. https://doi.org/10.3846/jbem.2018.1477

Acharya, S. (2019). Beyond Learning Outcomes: Impact of Organizational Flexibility on Strategic Performance Measures of Commercial E-Learning Providers. Global Journal of Flexible Systems Management, 20(1), 31-41. https://doi.org/10.1007/s40171-018-0199-3

Alimehr, M., Tahmasebi, M. J., Rastak, L., Moradi, A., Naghavi, H., Gahan, R., and Kord, A. (2019). The Relationship between Knowledge Management and Organizational Health among Employees of Health Centers in Ahvaz Jundishapur University. Journal of Research in Medical and Dental Science, 7(2), 137-144. Retrieved from https://cutt.ly/NYIZBxp

Ariza, D. (2017). Efectividad de la gestión de los proyectos: una perspectiva constructivista. Obras y Proyectos, 22, 75-85. https://doi.org/http://dx.doi.org/10.4067/S0718-28132017000200075

Ashraf, G., and Abd, S. bte. (2012). A review on the models of organizational effectiveness: A look at Cameron's model in higher education. International Education Studies, 5(2), 80-87. https://doi.org/10.5539/ies.v5n2p80

Bandera, C., Keshtkar, F., Bartolacci, M. R., Neerudu, S., and Passerini, K. (2017). Knowledge management and the entrepreneur: Insights from Ikujiro Nonaka's Dynamic Knowledge Creation model (SECl). International Journal of Innovation Studies, 1(3), 163-174. https://doi.org/10.1016/j.jijs.2017.10.005

Bustinza, O., Vendrell-Herrero, F., Perez-Arostegui, M., and Parry, G. (2019). Technological capabilities, resilience capabilities and organizational effectiveness. International Journal of Human Resource Management, 30(8), 1370-1392. https://doi.org/10.1080/09585192.2016.1216878

Camue, A., Carballal, E., and Toscano, D. F. (2017). Concepciones teóricas sobre la efectividad organizacional y su evaluación en las universidades. Cofin Habana, 11(2), 136-152. https://doi.org/https://doi.org/S2073-60612017000200010

Corrêa, C., Falsarella, O., and Cibele, S. (2016). Gestão do conhecimento : um estudo de modelos e sua relação com a inovação nas organizações. Perspectivas Em Ciência Da Informação, 21(1), 97-118. https://doi.org/https://doi.org/10.1590/1981$5344 / 2462$

Correia, P. M., Mendes, I., and Marques, N. (2018). Gestão Do Conhecimento E Da Inovação. Determinantes Da Competitividade Organizacional - Um Estudo De Caso De Uma Empresa De Consultoria Tecnológica. Revista Estudo \& Debate, 25(1), 192-215. https://doi.org/10.22410/issn.1983-036x.v25i1 a2018.1611

Fashami, C. S., and Babaei, M. (2017). A Behavioral Maturity Model to Establish Knowledge Management in an Organization. Engineering, Technology \& Applied Science Research, 7(3), 1725-1731. https://doi.org/10.48084/etasr.987

Gao, T., Chai, Y., and Liu, Y. (2018). A review of knowledge management about 
theoretical conception and designing approaches. International Journal of Crowd Science, 2(1), 42-51. https://doi.org/10.1108/ijcs-08-2017-0023

Kataria, A., Rastogi, R., and Garg, P. (2012). Organizational Effectiveness as a Function of Employee Engagement. South Asian Journal of Management, 20(4), 56-73. Retrieved from https://cutt.ly/pYIZ53Q

Khan, Z., and Vorley, T. (2017). Big data text analytics: an enabler of knowledge management. Journal of Knowledge Management, 21(1), 18-34. https://doi.org/10.1108/JKM-06-2015-0238

Koehler, F., Caetano, L., and Rogério, C. (2019). Understand the Dynamic Theory of Organizational Knowledge Creation; roots and future. International Journal for Innovation Education and Research, 7(9), 124-141. https://doi.org/10.31686/ijier.vol7.iss9.1715

Kumar, H. (2017). The Roles of Knowledge Management for the Development of Organizations Human Rights View project Sustainable Devwlopment View project The Roles of Knowledge Management for the Development of Organizations. Article $\square$ February, 2(2), 1-27. Retrieved from www.jsciachv.com

Marín, D. A., and Cuartas, J. C. (2019). Relationship between innovation and performance: Impact of competitive intensity and the organizational slack. $R A E$ Revista de Administracao de Empresas, 59(2), 95-107. https://doi.org/10.1590/S0034-759020190203

Mauricio, R., Pailaquilén, B., and Medina, E. (2019). Artículo Teórico. Revista San Gregorio, $35(4)$, https://doi.org/http://dx.doi.org/10.36097/rsan.v1i35.1109

198-207.

Passaillaigue, R., and Estrada, V. (2016). La gestión del conocimiento y el aprendizaje organizacional en instituciones de educación superior. GECONTEC: Revista Internacional de Gestión Del Conocimiento y La Tecnología, 4(2), 35-45. Retrieved from https://cutt.ly/WYIXpRk

Rehman, S., Mohamed, R., and Ayoup, H. (2019). The mediating role of organizational capabilities between organizational performance and its determinants. Journal of Global Entrepreneurship Research, 9(1). https://doi.org/10.1186/s40497-019-01555

Roza, R. H. (2020). Revisitando a Teoria da Criação do Conhecimento Organizacional. Revista Interamericana de Bibliotecología, 43(3), eRv2. https://doi.org/10.17533/udea.rib.v43n3erv2

Saad, G. Ben, Altheeb, S. Al, and Abbas, M. (2020). Knowledge Management Practices and Transformational Leadership Traits: Predicting Process Innovation in FMCG Industry. Propósitos $\quad$ y Representaciones, 8(SPE2). https://doi.org/10.20511/pyr2020.v8nspe2.662

Tang, H. (2017). A study of the effect of knowledge management on organizational culture and organizational effectiveness in medicine and health sciences. Eurasia Journal of Mathematics, Science and Technology Education, 13(6), 1831-1845. https://doi.org/10.12973/eurasia.2017.00700a

Velásquez, I. C. (2018). Gestión del conocimiento y la efectividad organizacional en el centro de operaciones Lima de la Empresa Ecocentury S.A.C Chorrillos - 2018. Retrieved from http://repositorio.autonoma.edu.pe/handle/AUTONOMA/684

Victer, R. S. (2020). Connectivity knowledge and the degree of structural formalization: a contribution to a contingency theory of organizational capability. Journal of Organization Design, 9(1). https://doi.org/10.1186/s41469-020-0068-3

Xavier, L. A. O. P., Oliveira, M., and Teixeira, E. K. (2012). Teorias utilizadas nas investigações sobre gestão do conhecimento. RISTI - Revista Iberica de Sistemas e Tecnologias de Informacao, 12(10), 1-17. https://doi.org/10.4304/risti.10.1-18

Zelenkov, Y. (2018). The impact of knowledge management and change readiness on the effectiveness of Russian private and state-owned organizations. Communications in Computer and Information Science, 877(4), 251-262. https://doi.org/10.1007/978-3-319-95204-8_22

Zeña, C. N., and Arévalo, J. A. (2016). Modelo de gestión del conocimiento para mejorar 
la calidad de investigación científica en universidades del Perú. UCV-HACER: Revista de Investigación y Cultura, 5(2), 76-83. https://doi.org/https://doi.org/6090815

Zidane, Y. J. T., and Olsson, N. O. E. (2017). Defining project efficiency, effectiveness and efficacy. International Journal of Managing Projects in Business, 10(3), 621641. https://doi.org/10.1108/lJMPB-10-2016-0085 